\title{
Wireless Landslide Geological Hazard Monitoring and Warning System
}

\author{
Chen Ban ${ }^{1, a}$, Chen Xiaohui ${ }^{2, b}$, Jiang Jirong ${ }^{3, c}$ and Wang Baoqiang ${ }^{4, d}$ \\ ${ }^{1}$ Computer Science and Engineering School, Jiangsu University of Science and Technology, \\ Zhenjiang, Jiangsu Province, P.R.China, 212003 \\ ${ }^{2,3,4}$ School of Automation, Nanjing University of Telecommunications and Posts, Nanjing, Jiangsu \\ Province, P.R.China, 210046 \\ aetta36@qq.com, bchenxh@njupt.edu.cn, cjjr10293@163.com, d15951961116@163.com
}

\begin{abstract}
Keywords: Landslide; Monitoring and warning; Wireless broadband communication; The internet of things
\end{abstract}

\begin{abstract}
Landslide geological hazard may cause great injuries and lost due to its crypticity and destroy. Advanced internet of things and wireless broadband communication techniques were adopted to realize remote wireless geological hazard monitoring and warning system and replace traditional manual work based monitoring method. Frames of hardware and software of the system were presented. Demonstration system was implemented at trouble spots in Wuxi. The results show that the monitoring system could collect real-time data of the monitored spots remotely, which provided necessary information for the geological hazard evaluation and warning.
\end{abstract}

\section{Introduction}

Our country has complex geological environment and geological hazards happen frequently. In 2013, there were 15403 geological hazards nationwide, causing a total of 481 people killed and 188 missing and direct economic loss of 10.2 billion yuan, which was learned from the Ministry of Land. Compared with 2012, the year of 2013 saw a 7.5\% increase on the number of geological hazards, a $78.4 \%$ increase on the number of the death and the missing and a $92.2 \%$ increase on the direct economic loss. For a long time, China has established monitoring network basically covering hilly mountainous counties in the whole country, which effectively prevented thousands of hazards each year. However, existing monitoring tools work in manual collection methods, technical solutions feature delayed data collection and incomplete information coverage. Even if the existing field automatic monitoring is limited to a few independent demonstration areas, the region monitoring devices do not form a real-time dynamic monitoring system. It is difficult to meet the needs of the unexpected landslide warning and rapid emergency.

Wireless sensor network technology extends sensing antennae of the sensors to achieve non-contact transmission, real-time monitoring, collaborating, localization decisions of target state information and ubiquitous access with the Internet and mobile communication networks, greatly improving the timeliness, reliability and flexibility of information acquisition. Based on sensor network technology and combined with broadband mobile communication technology, hazard monitoring demonstration zones of different geological environment and climatic conditions were chosen, solving the key technologies of monitoring and early warning for landslide and debris flow and establishing monitoring and early warning system for real-time dynamic data monitoring. The prediction model for landslides was built and timely capture of precursor information of the 
landslide hazard would provide technological support for prevention and cure for the landslide hazard and ensure stable and healthy development of national economy.

In this paper, typical landslide geological hazards were studied as monitoring objects. The remote monitoring system based on wireless $4 \mathrm{G}$ broadband communication network was researched. System was designed and field functional verification was completed, achieving the implementation of the monitoring and geological hazard warning.

\section{Formation of geological hazards landslides and monitoring principles}

Landslide is natural phenomenon that rock soil on the slope moves down as a whole or dispersedly along a certain weak belt of surface by the impact of river erosion, groundwater activity, earthquakes, artificial slope cutting and other factors as a result of its gravity[1-2]. The internal conditions of landslide are related to the nature, structure, and composition of rock-soil. Firstly, different rock-soil compositions vary in the shear strength, wind and water resistant to erosion; secondly, as far as the construction and structure of the rock-soil is concerned, the tendency in rock (soil) layers, the fault plane, degree of weathering, cracks and so on produces different effects on the phylogenetic relationship of the landslide; thirdly, the stability of the slope is affected by the section size of the slope. Figure 1 shows the picture of a kind of landslide geological hazard.

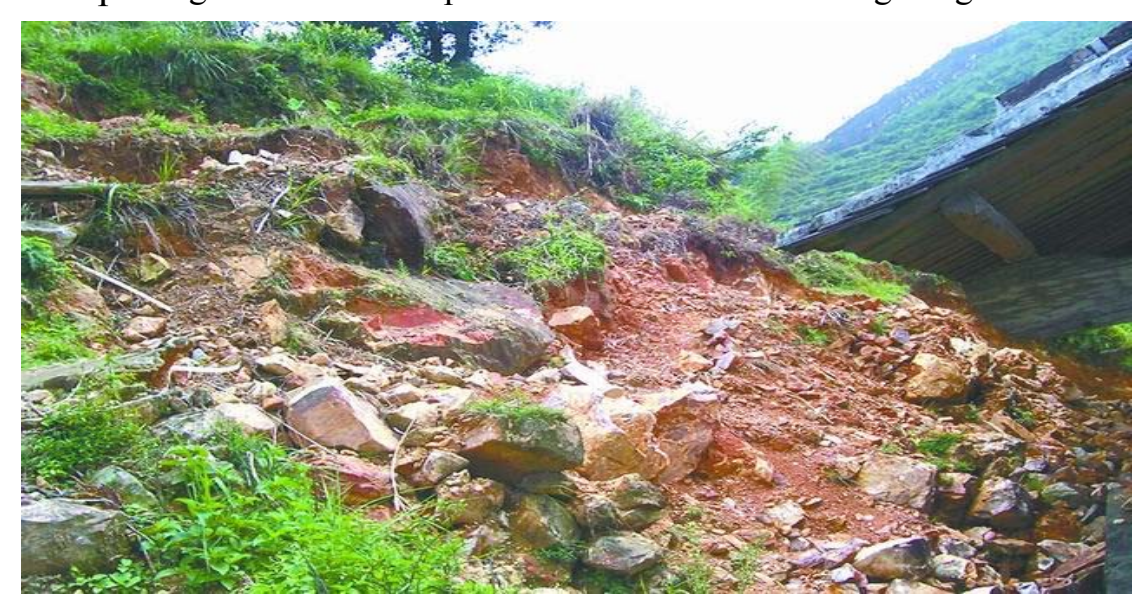

Fig.1, Picture of a kind of landslide hazard

The external conditions of landslide development are related to water effect, unreasonable excavation loading, vibration, mining on slope surface, and the former two functions are predominant. A survey showed that $90 \%$ of the landslides were water-related. Water comes in the form of atmospheric precipitation, surface water, groundwater, irrigation seepage, high pool and drains and the like.

According to the formation mechanism of these landslides, several important data of landslides formation would be monitored, including wind speed and direction, rainfall, soil moisture, soil displacement and surface pressure after sliding, while a video camera to capture the image information was used. Data processing was done to determine the soil cracks and other information. Thus, sensors we chose included sensors of wind speed, wind direction, rainfall, humidity, displacement, soil pressure and so on, constituting the front monitoring system. The monitoring system could be set according to the circumstances of landslide hazard monitoring and warning spot, and data were preprocessed and temporarily stored. Detection data were sent via the monitoring system. Monitoring data were converged through any WiFi gateway access, and reached a certain $3 \mathrm{G} / 4 \mathrm{G}$ gateway through the convergence network relay. $3 \mathrm{G} / 4 \mathrm{G}$ gateway could be used to send data converged through WiFi to the monitoring center.

To make better use of mobile communication infrastructure, the system applied the $3 \mathrm{G} / 4 \mathrm{G}$ network for data exchange with the remote geological hazard monitoring central station. $3 \mathrm{G} / 4 \mathrm{G}$ network features wide coverage 
and high data transmission rate, basically meeting the needs of current transmission of monitoring sensor and multimedia data and significantly reducing the development and deployment costs, which was a good choice for the convergence transmission of geological hazard monitoring data.

\section{Design and implementation of wireless monitoring and early warning system}

System solution structure is shown in Figure 2, according to the typical mechanism of the formation and evolution of geological hazards, wireless monitoring and early warning system was constructed combining the technologies of multi-sensors data fusion, broadband mobile communications, image processing and landslide monitoring and early warning to meet the real demand for geological hazard monitoring. In accordance with the foregoing analysis, the front monitoring station worked through the real-time collection of information of rainfall, humidity, wind speed, wind direction, displacement, earth pressure and rock (soil) evolution-related physical information, and acquisition of the overall mountain state video through the network HD camera. The local rock slide, the occurrence and development of cracks were discovered by the change of video data[3-5], The real-time data was sent to the base station via mobile 4G broadband communication router and eventually transferred to the geological hazard monitoring center, forming the final monitoring results applied to the alarm.

For relatively complex geological compositions of different monitoring locations, initially we used threshold alarm mechanism to achieve monitoring and early warning of emergency situations in the monitoring spots. Warning parameters set mainly included soil pressure, soil displacement, rainfall and wind speed. When the monitored parameters exceeded the thresholds, condition occurred and the monitoring system gave instructions to alert staff to the situation of the monitoring spot. And video images on the scene could be used for further analysis and judgment.

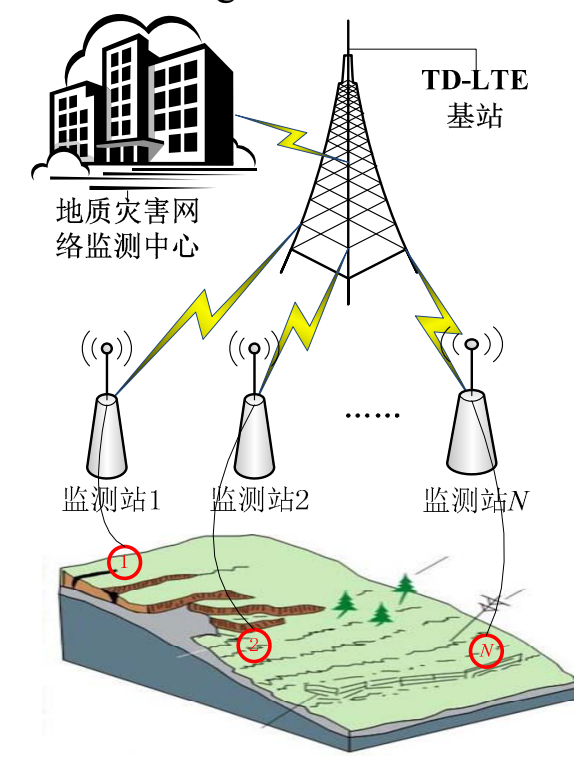

(a) System framework

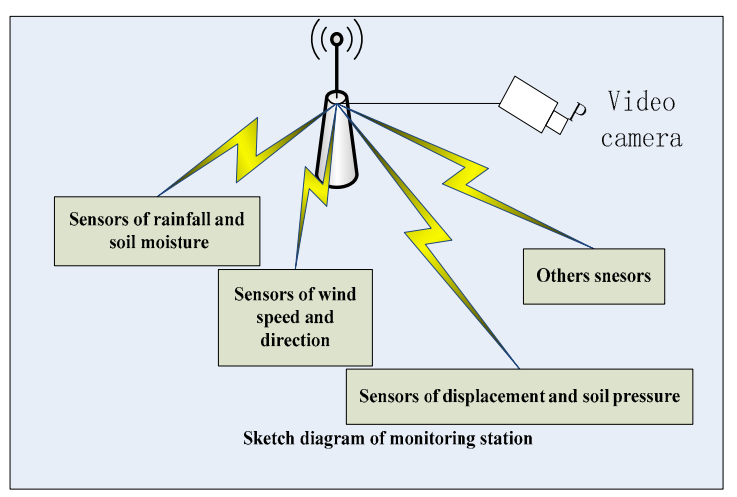

(b) The on-site hardware

Fig. 2, landslide wireless monitoring and warning system structure

Earth pressure load meter: the vibrating-wire pressure cell is a measuring sensor for earth pressure. It is manufactured according to the tension string theory. With frequency as the output signal, it features strong anti-interference ability and low long-distance transmission error. There is a built-in temperature sensor for the temperature correction as a result of external temperature change. And there is a computing chip for automatic conversion of measured data and direct physical output to reduce errors of manual conversion. All components go through rigorous testing and aging screening, especially in high-temperature stress relief test to enhance the stability and 
reliability of the chord. Additional three-anti treatment is to ensure high survival rate in the harsh environment.

Displacement meter: the single-point settlement meter consists of displacement meter, anchor head, flange settlement plate, length rod and other components, fit for measuring the deformation displacement of soil settlement between the anchor head and the disk. It can conduct long-term monitoring and automated measurement. Its principle lies in the measured structure deformation will drive the rod via anchor head of digital displacement meter, the rod would pull the displacement of displacement meter passed to vibrating wire displacement meter and turned into vibrating wire stress changes, thereby changing the vibration frequency. Electromagnetic coil stimulates the vibration wire and measures the vibration frequency. The signal is transmitted to the reading device via cable. And the amount of deformation of the test structure could be calculated and simultaneously the temperature value is obtained of the embedded point.

Soil moisture sensor: it is based on dielectric theory and application of frequency-domain measurement technology to accurately measure the volumetric water content of soil and other porous media. It can be integrated with the greenhouse environment monitoring system, the soil moisture collection system and the automatic irrigation control system to achieve long-term dynamic and continuous monitoring of moisture.

Rainfall sensor: the tipping bucket rainfall sensor is applied to measure the natural rainfall, while converting rainfall to digital output to meet the requirement of information transmission, processing, recording, display and the like.

Wind speed sensor: using three-cup wind speed sensor, wind speed sensor consists of housing, the cups and the circuit module. Cups and wind sensor housing are made of aluminum alloy material by the use of special precision molds die-casting process, so the dimensional tolerance is low and the surface accuracy is high. Its internal circuit is protectively treated and The entire sensor features high strength, strong weather resistance, anti-corrosion and water resistance.

Wind sensor: it is to measure horizontal direction of wind.

The monitoring system software includes mainly the following functions:

Real-time data monitoring: The parameters of wind speed, wind direction, rainfall, soil pressure, displacement and soil moisture were grasped timely and sent back to the network control center.

Alarm function: Mainly for parameters of soil pressure, rainfall, wind speed and soil displacement, when the monitoring data reached a pre-set threshold, the red reminder appeared on the host interface.

Data query: real-time data collected would be automatically saved to the Access database, the software provides a database query module and corresponding data is acquired according to the time of the query.

The main process of the monitoring and early warning system is shown in Figure 3 below.

The software system was developed using LabVIEW graphical programming environment. Figure 4 shows the core wireless data acquisition software process of the monitoring and early warning system. 


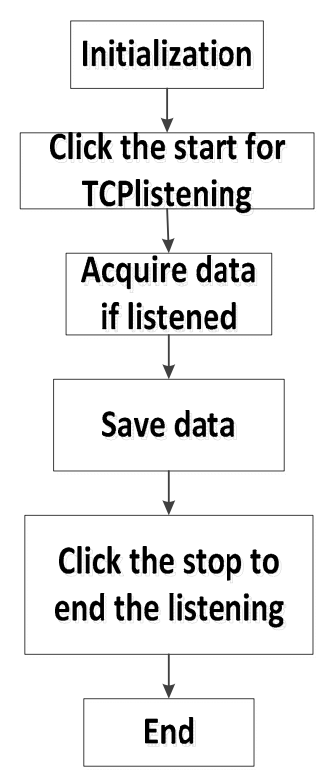

Fig.3, The main process of the monitoring and early warning system

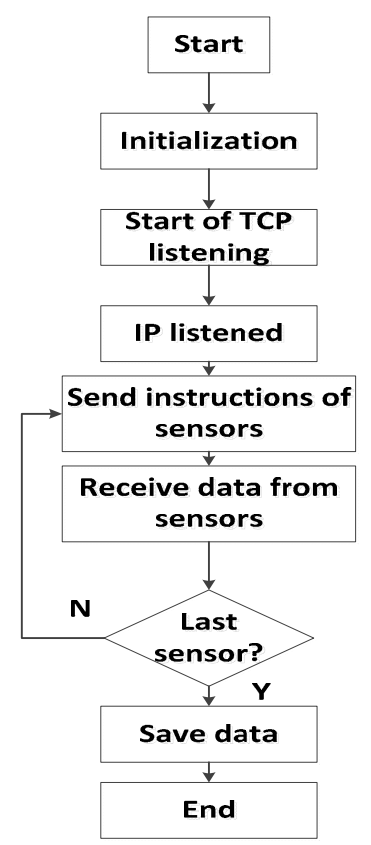

Fig. 4, Acquisition software process of the monitoring and early warning system

\section{Construction and operation of the system}

We selected typical geological hazard-prone zones in Wuxi hilly areas, designed and implemented a landslide wireless monitoring and early warning system. The selected sites included An-yang mountain geological hazard monitoring spot, Hengshan Temple geological hazard monitoring spot in Xuelang Mountain, Shanshui Road East monitoring spot and geological science garden in Xihui Park. Figure 5 shows the arrangement of monitoring devices in a monitoring spot.

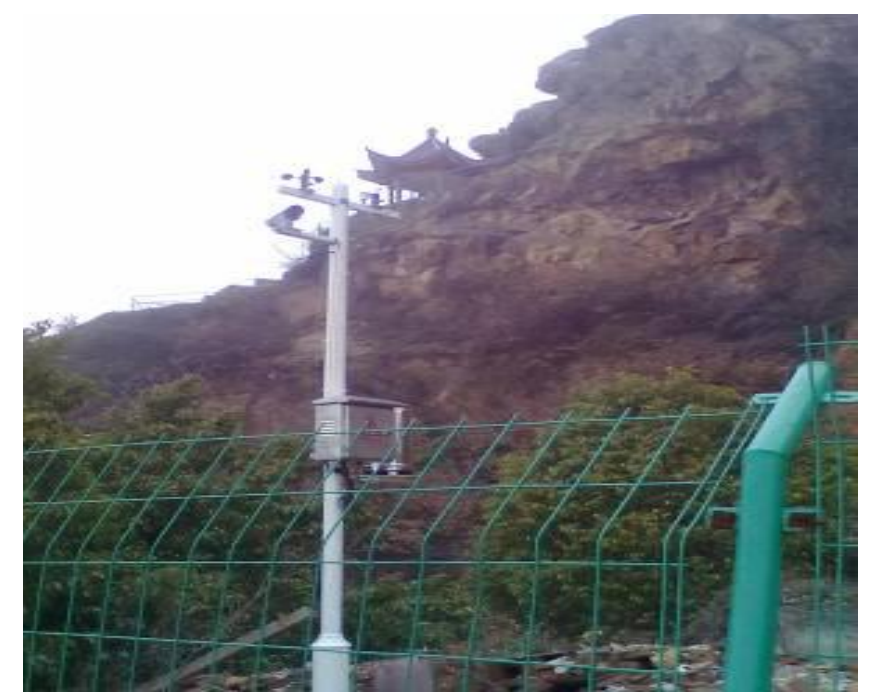

Fig. 5, Site of An-yang Mountain

Figure 6 shows the main interface of monitoring system software and data acquisition interface of An-yang Mount. Through test, the system remotely got the data from the on-site sensors, including video data, via advanced $4 \mathrm{G}$ wireless broadband communication technology. Some monitoring data was basically consistent with data from the local weather forecasts. The system was operated stably. Due to gradual changes of monitored rock and soil physical parameters and in order to save the network traffic, the data was acquired at regular time. Real time acquisition could be realized through interface control by users. 


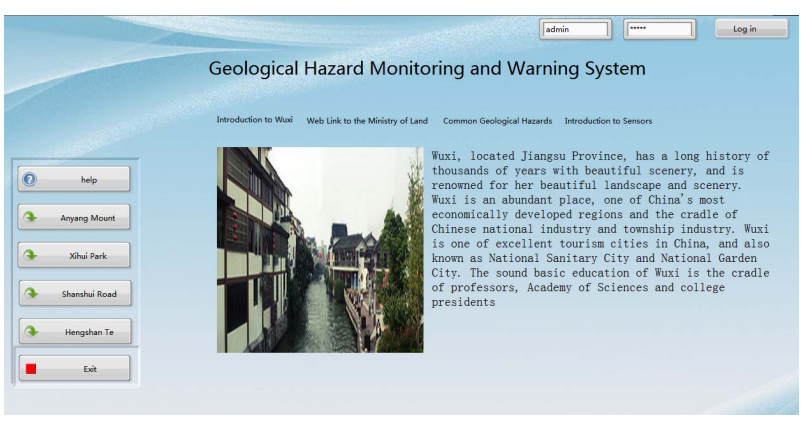

(a) Main display interface

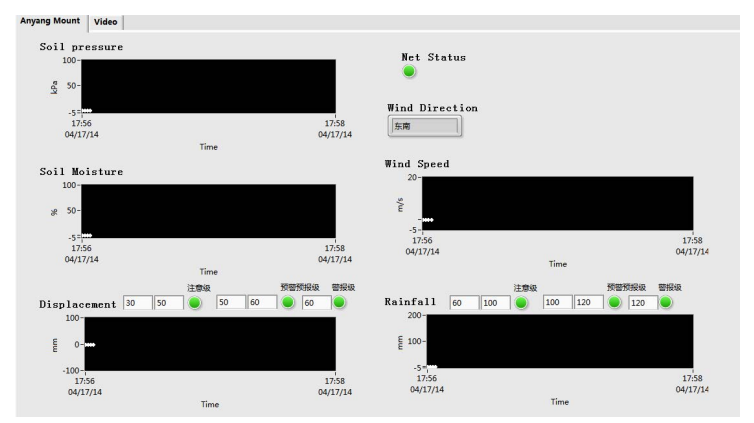

(b) Data acquisition display interface of An-yang Mount

Fig. 6, Main interface of software system and interface of data acquisition of An-yang Mount

\section{Conclusion}

In this paper, internet of things technology and mobile communication technology were integrated. We studied technologies of internet of things and mobile communications and applied them to geological hazard monitoring, designed and developed wireless landslide geological hazard monitoring and early warning system. Data from the on-site sensors and images from video surveillance equipment were sent timely to the monitoring center with the use of mobile communication technology. System software was designed for data processing and ultimately information of the corresponding geological hazards suggestions was obtained. Through field monitoring and operation, the system can achieve a stable performance of remotely real-time data acquisition of high accuracy and provide a better solution for geological hazard monitoring and early warning.

\section{References}

[1] F.J.Wang: Mining Engineering (Chinese), Vol.9(3) (2011), p.21-22

[2] C.X.Mao, X.B.Duan: Journal of Geotechnical Engineering (Chinese), Vol. 34(11) (2012), p. 2148-2151

[3] B.Xu: Computer Measurement and Control (Chinese), Vol. 21(9) (2013), p, 2409-2411

[4] Y.D.Zhou: Railway Signaling and Communication (Chinese), Vol. 47(4) (2011), p. 77-80

[5] X.Zh.Liu, J.X.Zeng, B.Tian: Computer Applications and Software (Chinese), Vol. 30(12) (2013), p. 56-59 